\title{
THE MINIMUM MATCHING ENERGY OF BICYCLIC GRAPHS WITH GIVEN GIRTH
}

\author{
HONG-HAI LI AND LI ZOU
}

\begin{abstract}
The matching energy of a graph was introduced by Gutman and Wagner in 2012 and defined as the sum of the absolute values of zeros of its matching polynomial. Let $\theta(r, s, t)$ be the graph obtained by fusing two triples of pendant vertices of three paths $P_{r+2}, P_{s+2}$ and $P_{t+2}$ to two vertices. The graph obtained by identifying the center of the star $S_{n-g}$ with the degree 3 vertex $u$ of $\theta(1, g-3,1)$ is denoted by $S_{n-g}(u) \theta(1, g-3,1)$. In this paper, we show that, $S_{n-g}(u) \theta(1, g-3,1)$ has minimum matching energy among all bicyclic graphs with order $n$ and girth $g$.
\end{abstract}

1. Introduction. All graphs in this paper are finite, simple and nondirected. Let $G=(V, E)$ be such a graph with order $|V|=n$ and size $|E|=m$. In a graph a matching is a set of pairwise nonadjacent edges, and we denote the number of $k$-matchings of $G$ by $m_{k}(G)$. Note that $m_{1}(G)=m$ and $m_{k}(G)=0$ for $k>\lfloor n / 2\rfloor$. It is both consistent and convenient to define $m_{0}(G)=1$. The matching polynomial of $G$ is defined as

$$
\alpha(G, x)=\sum_{k \geq 0}(-1)^{k} m_{k}(G) x^{n-2 k} .
$$

All the zeros of $\alpha(G, x)$ are real-valued and the theory of matching polynomials is well elaborated in $[3,5]$.

Recently, Gutman and Wagner [7] introduced the matching energy of a graph $G$, denoted by $\operatorname{ME}(G)$ and defined as

2010 AMS Mathematics subject classification. Primary 05C35, 05C50.

Keywords and phrases. Bicyclic graph, matching energy, girth.

The first author is supported by National Natural Science Foundation of China, (grant Nos. 11201198, 11561032), the Scientific Funds of the Education Department of Jiangxi Province (grant No. GJJ150345), and the Sponsored Program for Cultivating Youths of Outstanding Ability in Jiangxi Normal University. The first author is the corresponding author.

Received by the editors on September 20, 2014. 


$$
\operatorname{ME}(G)=\frac{2}{\pi} \int_{0}^{\infty} \frac{1}{x^{2}} \ln \left[\sum_{k \geq 0} m_{k}(G) x^{2 k}\right] d x,
$$

which coincides with the Coulson-type integral formula for the energy which has been studied extensively (see an excellent monograph [13] and $[\mathbf{1 4}]$ and the references therein for recent advances), when the graph under consideration is a tree. As mentioned in [7], matching energy can also be defined in another form as follows.

Let $G$ be a simple graph, and let $\mu_{1}, \mu_{2}, \ldots, \mu_{n}$ be the zeros of its matching polynomial. Then

$$
\operatorname{ME}(G)=\sum_{i=1}^{n}\left|\mu_{i}\right| .
$$

The integral on the right side of equation (1.1) is increasing in all the coefficients $m_{k}(G)$. This means that if two graphs $G$ and $G^{\prime}$ satisfy $m_{k}(G) \leq m_{k}\left(G^{\prime}\right)$ for all $k \geq 1$, then $\operatorname{ME}(G) \leq \operatorname{ME}\left(G^{\prime}\right)$. If, in addition, $m_{k}(G)<m_{k}\left(G^{\prime}\right)$ for at least one $k$, then $\operatorname{ME}(G)<\operatorname{ME}\left(G^{\prime}\right)$. This then motivates the introduction of a quasi-order $\succeq$, defined by

$$
G \succeq H \Longleftrightarrow m_{k}(G) \geq m_{k}(H),
$$

for all nonnegative integers $k$. If $G \succeq H$ and there exists some $k$ such that $m_{k}(G)>m_{k}(H)$, then we write $G \succ H$. It is said that $G$ is $m$-greater than $H$ if $G \succeq H$ and strictly m-greater than $H$ if $G \succ H$. It is easy to see that

$$
G \succeq H \Longrightarrow \operatorname{ME}(G) \geq \operatorname{ME}(H)
$$

and

$$
G \succ H \Longrightarrow \operatorname{ME}(G)>\operatorname{ME}(H) .
$$

Initial work on matching energy of graphs is attributed to [7] and then followed by $\mathrm{Li}$ and Yan [16] who characterized the maximal connected graphs with given connectivity and chromatic numbers. The extremal graphs in connected bicyclic graphs were determined by $\mathrm{Ji}$, $\mathrm{Li}$ and Shi $[\mathbf{1 0}]$ and further by Chen, Liu and Shi $[\mathbf{1 , 2}]$ for unicyclic, bicyclic and tricyclic graphs. More generally, the minimal matching 
energy of $(m, n)$-graphs with a given matching number was obtained by Xu, Das and Zheng [19]. Huang, Kuang and Deng [9] characterized the extremal graph for a random polyphenyl chain. For more results, see $[\mathbf{1 1}, \mathbf{1 2}, \mathbf{1 7}]$ and there may be other results which are unknown to the authors.

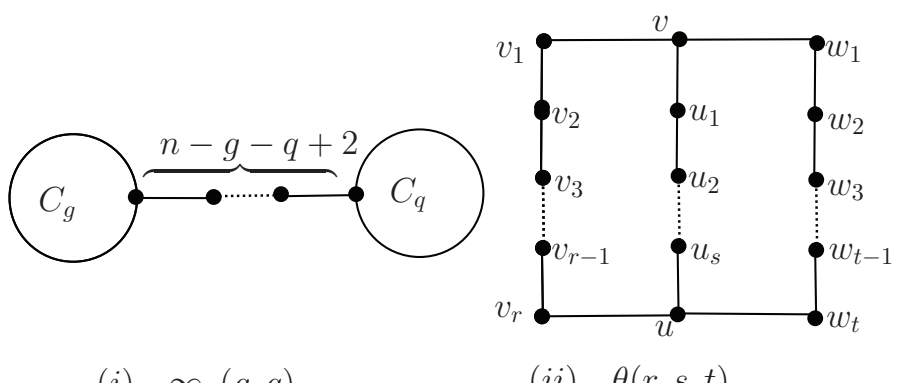

(i) $\infty_{n}(g, q)$

(ii) $\theta(r, s, t)$

FiguRE 1. Two types of braces: $\infty_{n}(g, q)$ and $\theta(r, s, t)$.

Denote the set of all connected bicyclic graphs with order $n$ and girth $g$ by $\mathscr{B}_{n, g}$. We now define two special classes of bicyclic graphs. Let $\infty_{n}(g, q)$ denote the graph obtained by the coalescence of two end vertices of a path $P_{n-g-q+2}$ with one vertex of two cycles $C_{g}$ and $C_{q}$, respectively, and $\theta(r, s, t)$ the graph obtained by fusing two triples of pendant vertices of three paths $P_{r+2}, P_{s+2}$ and $P_{t+2}$ to two vertices, as given in Figure 1. The distance of two cycles $C_{g}$ and $C_{q}$ in $G$ is defined as

$$
d_{G}\left(C_{g}, C_{q}\right)=\min \left\{d_{G}(x, y) \mid x \in V\left(C_{g}\right), y \in V\left(C_{q}\right)\right\},
$$

sometimes written as $d_{G}$ for short. Note that $d_{G}\left(C_{g}, C_{q}\right)=0$ if $C_{g}$ and $C_{q}$ have a common vertex, e.g., for $G=\infty_{n}(g, q)$ such that $q=n-g+1$, and in this case, $\infty_{n}(g, q)$ with $q=n-g+1$ is simply written as $\infty(g, q)$ for convenience. Clearly, any bicyclic graph must contain either graph (i) or (ii) in Figure 1 as an induced subgraph, called its brace. Then the set $\mathscr{B}_{n, g}$ can be partitioned into two subsets $\mathscr{B}_{n, g}^{1}$ and $\mathscr{B}_{n, g}^{2}$, where $\mathscr{B}_{n, g}^{1}$ is the set of all bicyclic graphs which contain a brace of the form $\infty_{n}(g, q)$, and $\mathscr{B}_{n, g}^{2}$ is the set of all bicyclic graphs which contain a brace of the form $\theta(r, s, t)$. 
There have been some papers on characterizing the minimal Hosoya index of graphs, see $[4, \mathbf{1 8}]$. In this paper, minimal graphs in $\mathscr{B}_{n, g}^{i}$ for $i=1,2$, are determined respectively and, by comparing them, we show that $S_{n-g}(u) \theta(1, g-3,1)$, obtained by identifying the center of the star $S_{n-g}$ with a vertex $u$ of degree 3 in $\theta(1, g-3,1)$, has minimum matching energy among all bicyclic graphs in $\mathscr{B}_{n, g}$.

2. Preliminaries. In this section, we shall present some basic results which will be used in the proof of our main results.

Given a graph $G$ and an edge $u v$ of $G$, we denote by $G-u v$ (respectively $G-v$ ) the graph obtained from $G$ by deleting the edge $u v$ (respectively the vertex $v$ and edges incident to it).

Lemma 2.1 ([10]). If $u, v$ are adjacent vertices of $G$, then

$$
m_{k}(G)=m_{k}(G-u v)+m_{k-1}(G-u-v),
$$

for all nonnegative integers $k$.

Let $G(v) S_{t+1}$ (or $S_{t+1}(v) G$ ) denote the graph obtained by identifying the vertex $v$ of a graph $G$ with the center of the star $S_{t+1}$, as given in Figure 2.

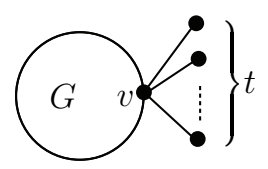

Figure 2. $G(v) S_{t+1}$.

Note. Consider the graph in Figure 2.

- $m_{k}(G)$ is the number of $k$-matchings that do not contain any of the edges of $S_{t+1}$.

- $t m_{k-1}(G-v)$ is the number of $k$-matchings that contain one of these edges, as there are $t$ choices for these edges in $S_{t+1}$.

Consequently, we have what follows without giving a formal proof. 
Lemma 2.2. Let $G$ be a graph, and let $v$ be a vertex of $G$. Then, $m_{k}\left(G(v) S_{t+1}\right)=m_{k}(G)+t m_{k-1}(G-v)$.

Recall a result in [13], which establishes the order of the union of two paths with a given number of vertices according to the quasi-order as stated in the introduction.

Lemma $2.3([\mathbf{1 3}])$. Let $n=4 k, 4 k+1,4 k+2$ or $4 k+3$. Then,

$$
\begin{aligned}
P_{n} & \succ P_{2} \cup P_{n-2} \succ P_{4} \cup P_{n-4} \succ \cdots \succ P_{2 k} \cup P_{n-2 k} \succ P_{2 k+1} \cup P_{n-2 k-1} \\
& \succ P_{2 k-1} \cup P_{n-2 k+1} \succ \cdots \succ P_{3} \cup P_{n-3} \succ P_{1} \cup P_{n-1} .
\end{aligned}
$$

Lemma 2.4 ([6]). If $G_{1} \succ G_{2}$, then $G_{1} \cup H \succ G_{2} \cup H$, where $H$ is an arbitrary graph.

Applying Lemma 2.4, we can generalize Lemma 2.3 to the following form, the union of three paths.

Lemma 2.5. Let $r, s, t$ be nonnegative integers with $r \leq s-2$. If $r$ is even, then

$$
\begin{aligned}
P_{r-2} \cup P_{s+2} \cup P_{t} & \succ P_{r} \cup P_{s} \cup P_{t} \succ P_{r+1} \cup P_{s-1} \cup P_{t} \\
& \succ P_{r-1} \cup P_{s+1} \cup P_{t} .
\end{aligned}
$$

Lemma 2.6 ([7]). Suppose that $G$ is a connected graph and $T$ an induced subgraph of $G$ such that $T$ is a tree and is connected to the rest of $G$ only by a cut vertex $v$. If $T$ is replaced by a star of the same order and centered at $v$, then the matching energy decreases (unless $T$ is already such a star). If $T$ is replaced by a path with one end at $v$, then the matching energy increases (unless $T$ is already such a path).

Recall the definition of a generalized $\pi$-transform in [15]. We say $Q$ is a branch of a connected graph $G$ with root $u$ if $Q$ is a connected induced subgraph of $G$ for which $u$ is the only vertex in $Q$ that has a neighbor not in $Q$. Let $P$ and $Q$ be branches of a component of a graph $G$ with a common root $u_{0}$, which is also their only common vertex. Assume that $P$ is a path and $u_{0}$ has at least one neighbor in $G$ that does not lie on $P$ or $Q$. Form a graph from $G$ by relocating 
the branch $Q$ from $u_{0}$ to $v$ where $v$ is the other end vertex of the path $P$ (by deleting edges $u_{0} w$ and adding new edges $v w$ for every vertex $w$ in $Q$ adjacent to $u_{0}$ ). We refer to the resulting graph as a generalized $\pi$-transform of $G$.

Theorem 2.7 ([15]). If $G^{\prime}$ is a generalized $\pi$-transform of $G$, then $G^{\prime} \succ G$ and so $\operatorname{ME}\left(G^{\prime}\right)>\operatorname{ME}(G)$.

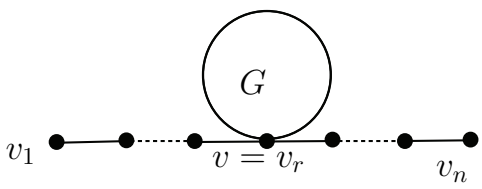

(i) $P_{n}\left(v_{r}, v\right) G$

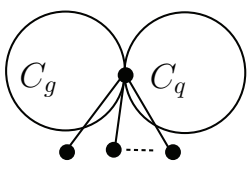

(ii) $S_{n}(g, q)$

Figure 3.

Let $P_{n}\left(v_{r}, v\right) G$ denote the graph obtained by identifying the vertex $v_{r}$ of $P_{n}$ with the vertex $v$ of $G$ (see Figure 3 (i)). For convenience, we use $P_{n}(v) G$ (or $G(v) P_{n}$ ) to stand for $P_{n}\left(v_{1}, v\right) G$. Note that $P_{n}\left(v_{r}, v\right) G$ and $P_{r}(v) G(v) P_{n-r+1}$ are isomorphic.

Theorem 2.8 ([8]). If $v$ is an arbitrary vertex of the graph $G$, then for $n=4 k+i, i \in\{-1,0,1,2\}, k \geq 1$,

$$
\begin{aligned}
P_{n}\left(v_{1}, v\right) G & \succ P_{n}\left(v_{3}, v\right) G \succ \cdots \succ P_{n}\left(v_{2 k+1}, v\right) G \succ P_{n}\left(v_{2 k}, v\right) G \\
& \succ P_{n}\left(v_{2 k-2}, v\right) G \succ \cdots \succ P_{n}\left(v_{2}, v\right) G .
\end{aligned}
$$

Let $G$ be an arbitrary graph with a specified vertex $v$. The graph obtained from $G$ is denoted by $\widehat{\mathcal{G}}_{i}$ for $i=1,2, \ldots, n-1$ (as given in Figure 4), by adding $n-1$ new vertices to $G$ in the following way. Attach $i-1$ pendant edges and a pendant path of length $n-i$ at $v$. By Theorem 2.8, we easily obtain the following.

Lemma 2.9. $\widehat{\mathcal{G}}_{1} \succ \widehat{\mathcal{G}}_{2} \succ \cdots \succ \widehat{\mathcal{G}}_{n-1}$.

Proof. $\widehat{\mathcal{G}}_{1} \succ \widehat{\mathcal{G}_{2}}$ follows immediately from Theorem 2.8 , as $\widehat{\mathcal{G}}_{1} \cong$ $P_{n}\left(v_{1}, v\right) G$ and $\widehat{\mathcal{G}}_{2} \cong P_{n}\left(v_{2}, v\right) G$. In fact, other cases can be verified 


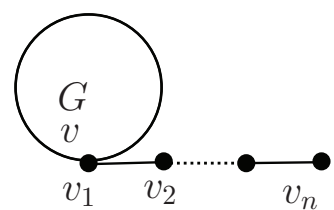

$\mathcal{G}_{1}$

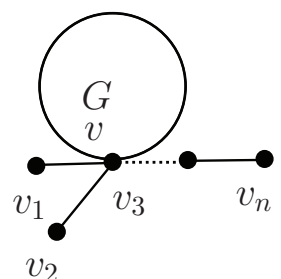

$\mathcal{G}_{3}$

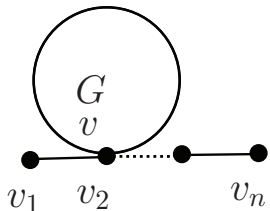

$\mathcal{G}_{2}$

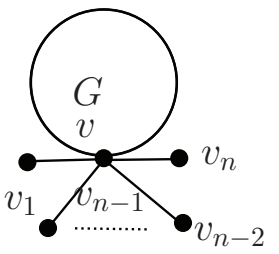

$v_{2}$

$\mathcal{G}_{n-1}$

Figure 4.

in the same way. Note that if we denote the graph $G(v) S_{i}$ by $H$, then $\widehat{\mathcal{G}}_{i} \cong P_{n-i+1}\left(v_{1}, v\right) H$ and $\widehat{\mathcal{G}}_{i+1} \cong P_{n-i+1}\left(v_{2}, v\right) H$.

Let $S_{n}(g, q)$ be the graph in $\mathscr{B}_{n, q}^{1}$ with $n+1-(g+q)$ pendant edges attached at the common vertex of $C_{g}$ and $C_{q}$ (see Figure 3 (ii)).

Theorem 2.10. $S_{n}(g, q) \succeq S_{n}(g, g)$ with equality if and only if $g=q$.

Proof. Let $u\left(u^{\prime}\right.$, respectively) be the common vertex of $C_{g}$ and $C_{q}\left(C_{g}\right.$, respectively) in $S_{n}(g, q)\left(S_{n}(g, g)\right.$, respectively), and $u_{1} u_{2}$ $\left(u_{1}^{\prime} u_{2}^{\prime}\right.$, respectively) an edge of $C_{q}\left(C_{g}\right.$, respectively) such that $u_{1}\left(u_{1}^{\prime}\right.$, respectively) is adjacent to $u$ ( $u^{\prime}$, respectively). By Lemma 2.1, we have

$$
m_{k}\left(S_{n}(g, q)\right)=m_{k}\left(S_{n}(g, q)-u_{1} u_{2}\right)+m_{k-1}\left(S_{n}(g, q)-u_{1}-u_{2}\right)
$$

and

$$
m_{k}\left(S_{n}(g, g)\right)=m_{k}\left(S_{n}(g, g)-u_{1}^{\prime} u_{2}^{\prime}\right)+m_{k-1}\left(S_{n}(g, g)-u_{1}^{\prime}-u_{2}^{\prime}\right) .
$$


Note that

$$
\begin{aligned}
& S_{n}(g, q)-u_{1} u_{2} \cong P_{q-1}(u) C_{g}(u) S_{n+3-g-q} \\
& S_{n}(g, g)-u_{1}^{\prime} u_{2}^{\prime} \cong P_{g-1}(u) C_{g}(u) S_{n+3-2 g} .
\end{aligned}
$$

If we denote the graph $P_{i-1}(u) C_{g}(u) S_{n+3-g-i}$ by $G_{i}$, then $S_{n}(g, q)-$ $u_{1} u_{2} \cong G_{q}$ and $S_{n}(g, g)-u_{1}^{\prime} u_{2}^{\prime} \cong G_{g}$. By Lemma 2.9, we have

$$
G_{q} \succ G_{q-1} \succ \cdots \succ G_{g} .
$$

Thus, $S_{n}(g, q)-u_{1} u_{2} \succeq S_{n}(g, g)-u_{1}^{\prime} u_{2}^{\prime}$ with equality if and only if $g=q$.

In the same way, we also get $S_{n}(g, g)-u_{1}-u_{2} \succeq S_{n}(g, g)-u_{1}^{\prime}-u_{2}^{\prime}$ with equality if and only if $g=q$. Therefore, $S_{n}(g, q) \succeq S_{n}(g, g)$ with equality if and only if $g=q$.

Theorem 2.11. If $t \geq 2$ and $r$ is even, then

$$
\begin{aligned}
\theta(r-2, s+2, t) & \succ \theta(r, s, t) \succ \theta(r+1, s-1, t) \\
& \succ \theta(r-1, s+1, t),
\end{aligned}
$$

where $r \leq s-2$ and $r+s+2=g$.

Proof. Let $G=\theta(r, s, t)$, which can be obtained by merging two triples of pendant vertices $u_{0}, v_{0}, w_{0}$ and $u_{r+1}, v_{s+1}, w_{t+1}$ of three paths

$$
P_{r+2}=u_{0} u_{1} \cdots u_{r} u_{r+1}, \quad P_{s+2}=v_{0} v_{1} \cdots v_{s} v_{s+1}
$$

and

$$
P_{t+2}=w_{0} w_{1} \cdots w_{t} w_{t+1},
$$

to two vertices, say $u$ and $v$, respectively. By Lemma 2.1, we have

$$
\begin{aligned}
m_{k}(G)= & m_{k}\left(G-u w_{1}\right)+m_{k-1}\left(G-u-w_{1}\right) \\
= & m_{k}\left(G-u w_{1}-v w_{t}\right)+m_{k-1}\left(G-u w_{1}-v-w_{t}\right) \\
& +m_{k-1}\left(G-u-w_{1}-v w_{t}\right) \\
& +m_{k-2}\left(G-u-w_{1}-v-w_{t}\right) \\
= & m_{k}\left(P_{t} \cup C_{g}\right)+2 m_{k-1}\left(P_{g-1} \cup P_{t-1}\right) \\
& +m_{k-2}\left(P_{r} \cup P_{s} \cup P_{t-2}\right) .
\end{aligned}
$$


For convenience, let $G_{1}=\theta(r-1, s+1, t), G_{2}=\theta(r-2, s+2, t)$ and $G_{3}=\theta(r+1, s-1, t)$. Applying the same method to the graphs $G_{1}$, $G_{2}$ and $G_{3}$, we get

$$
\begin{aligned}
m_{k}\left(G_{1}\right)= & m_{k}\left(P_{t} \cup C_{g}\right)+2 m_{k-1}\left(P_{g-1} \cup P_{t-1}\right) \\
& +m_{k-2}\left(P_{r-1} \cup P_{s+1} \cup P_{t-2}\right), \\
m_{k}\left(G_{2}\right)= & m_{k}\left(P_{t} \cup C_{g}\right)+2 m_{k-1}\left(P_{g-1} \cup P_{t-1}\right) \\
& +m_{k-2}\left(P_{r-2} \cup P_{s+2} \cup P_{t-2}\right), \\
m_{k}\left(G_{3}\right)= & m_{k}\left(P_{t} \cup C_{g}\right)+2 m_{k-1}\left(P_{g-1} \cup P_{t-1}\right) \\
& +m_{k-2}\left(P_{r+1} \cup P_{s-1} \cup P_{t-2}\right) .
\end{aligned}
$$

Thus,

$$
\begin{aligned}
m_{k}\left(G_{1}\right)-m_{k}(G)= & m_{k-2}\left(P_{r-1} \cup P_{s+1} \cup P_{t-2}\right) \\
& -m_{k-2}\left(P_{r} \cup P_{s} \cup P_{t-2}\right), \\
m_{k}\left(G_{2}\right)-m_{k}(G)= & m_{k-2}\left(P_{r-2} \cup P_{s+2} \cup P_{t-2}\right) \\
& -m_{k-2}\left(P_{r} \cup P_{s} \cup P_{t-2}\right), \\
m_{k}\left(G_{3}\right)-m_{k}\left(G_{1}\right)= & m_{k-2}\left(P_{r+1} \cup P_{s-1} \cup P_{t-2}\right) \\
& -m_{k-2}\left(P_{r-1} \cup P_{s+1} \cup P_{t-2}\right) .
\end{aligned}
$$

By Lemma 2.5, it follows directly that if $r \leq s-2$ and $r$ is even, then

$$
\begin{aligned}
P_{r-2} \cup P_{s+2} \cup P_{t} & \succ P_{r} \cup P_{s} \cup P_{t} \succ P_{r+1} \cup P_{s-1} \cup P_{t} \\
& \succ P_{r-1} \cup P_{s+1} \cup P_{t},
\end{aligned}
$$

and so assertion (2.1) holds.

As an immediate consequence, we have the following result.

Corollary 2.12. If $t \geq 2$, then $\theta(r, s, t) \succeq \theta(1, r+s-1, t)$ with equality if and only if $r=1$ or $s=1$.

Proof. Without loss of generality, assume that $r \leq s$. If $r=0$, by Theorem 2.11, we have either

$$
\theta(0, s, t)=\theta(r, s, t) \succ \theta(r+1, s-1, t)=\theta(1, s-1, t)
$$


when $s \geq 2$ or $s=1$ and, in this case, $\theta(0, s, t)$ is already of the form $\theta(1, r+s-1, t)$.

Now assume that $r \geq 1$. If $r$ is even, then $\theta(r, s, t) \succ \theta(r-1, s+1, t) \succ \theta(r-3, s+3, t) \succ \cdots \succ \theta(1, s+r-1, t)$. If $r$ is odd, then $\theta(r, s, t) \succ \theta(r-2, s+2, t) \succ \theta(r-4, s+4, t) \succ \cdots \succ \theta(1, s+r-1, t)$.

3. Main results. In this section, we first show that $S_{n}(g, g)$ has minimum matching energy in $\mathscr{B}_{n, g}^{1}$ and $S_{n-g}(u) \theta(1, g-3,1)$ is the minimal graph in $\mathscr{B}_{n, g}^{2}$. Further, by comparing these two graphs, we conclude that $S_{n-g}(u) \theta(1, g-3,1)$ is the unique graph with minimum matching energy in $\mathscr{B}_{n, g}$.

Theorem 3.1. For any graph $G \in \mathscr{B}_{n, g}^{1}$, we have $G \succeq S_{n}(g, g)$ with equality if and only if $G \cong S_{n}(g, g)$.

Proof. For any graph $G \in \mathscr{B}_{n, g}^{1}$, its brace must be of the form $\infty_{n}(g, q)$ for some $q \geq g$. By Lemma 2.6, if any tree branch is replaced by a star of equal order centered at the root, its matching energy strictly decreases unless the branch is already such a star. To show that $G \succeq S_{n}(g, g)$ for any $G \in \mathscr{B}_{n, g}^{1}$, it suffices to show it holds for such a graph $G$, all of whose tree branches are stars. We distinguish two cases according to the value of $d_{G}$, the distance between $C_{g}$ and $C_{q}$ in its brace.

Case 1. $d_{G}=0$. As above, if $G$ has $l$ tree branches, we can assume that these $l$ branches are stars. Without loss of generality, for $i=1, \ldots, l$, we will assume $G$ is the coalescence of the vertex $u_{i}$ in its brace $\infty(g, q)$ and the center of $S_{r_{i}+1}$. For convenience, we use the notation $G_{i}$ to denote such graphs recursively defined as follows. Let $G_{0}=\infty(g, q)$, and if $G_{i-1}$ is already defined, then $G_{i}$ is defined to be $G_{i-1}\left(u_{i}\right) S_{r_{i}+1}$. Note that $G_{l}=G$.

Applying Lemma 2.2 to $S_{n}(g, q)$, we have

$$
m_{k}\left(S_{n}(g, q)\right)=m_{k}(\infty(g, q))+(n+1-g-q) m_{k-1}\left(P_{g-1} \cup P_{q-1}\right) .
$$


Similarly applying Lemma 2.2 to $G$, we have

$$
\begin{aligned}
m_{k}(G) & =m_{k}\left(G_{l-1}\right)+r_{l} m_{k-1}\left(G_{l-1}-u_{l}\right) \\
& =m_{k}\left(G_{l-2}\right)+r_{l-1} m_{k-1}\left(G_{l-2}-u_{l-1}\right)+r_{l} m_{k-1}\left(G_{l-1}-u_{l}\right) \\
& =\cdots \\
& =m_{k}\left(G_{0}\right)+\sum_{i=1}^{l} r_{i} m_{k-1}\left(G_{i-1}-u_{i}\right),
\end{aligned}
$$

where

$$
\sum_{i=1}^{l} r_{i}=n+1-g-q
$$

First, $G_{0}=\infty(g, q)$. Second,

$$
G_{i-1}-u_{i} \succeq \infty(g, q)-u_{i} \succeq P_{g-1} \cup P_{q-1} \quad \text { for } i=1, \ldots, l,
$$

because each graph is a subgraph of the former. If $G \nsubseteq S_{n}(g, q)$, then for some $i, G_{i-1}-u_{i}$ has $P_{g-1} \cup P_{q-1}$ as a proper subgraph. Therefore, $G \succeq S_{n}(g, q)$ with equality if and only if $G \cong S_{n}(g, q)$. By Theorem 2.10, the assertion holds.

Case 2. $d_{G} \geq 1$. By Lemma 2.6, we can assume that all tree branches of $G$ are stars. Suppose $P=w_{1} w_{2} \cdots w_{t}$ is the unique path connecting two cycles $C_{g}$ and $C_{q}$ in $G$. We proceed by induction on the number of pendant vertices along the path $P$. If there are no pendant vertices along the path, by applying the inverse generalized $\pi$-transform to $G$, i.e., deleting all edges $w_{t} u$, where $u \in N\left(w_{t}\right) \backslash\left\{w_{t-1}\right\}$ and adding new edges $w_{1} u$, it becomes a graph in $\mathscr{B}_{n, g}^{1}$ with $d_{G}\left(C_{g}, C_{q}\right)=0$ and so, by Case $1, G \succeq S_{n}(g, g)$.

Now assume that there is at least a pendant edge $u v$ on the path $P$. By Lemma 2.1, we have $m_{k}(G)=m_{k}(G-u v)+m_{k-1}(G-u-v)$. By the induction hypothesis, $G-u v \succ S_{n-1}(g, q)$. Also, it is easy to see that $G-u-v$ has $P_{g-1} \cup P_{q-1}$ as its subgraph. Thus,

$$
\begin{aligned}
m_{k}(G) & =m_{k}(G-u v)+m_{k-1}(G-u-v) \\
& \geq m_{k}\left(S_{n-1}(g, q)\right)+m_{k-1}\left(P_{g-1} \cup P_{q-1}\right) \\
& =m_{k}\left(S_{n}(g, q)\right),
\end{aligned}
$$

and strict inequality holds for at least one $k$. Therefore, $G \succ S_{n}(g, q)$ and then $G \succ S_{n}(g, g)$ by Theorem 2.10 . 
Theorem 3.2. For any positive integers $r, s, t$ with $r+s+2=$ $g$, let $G_{1}, G_{1}^{\prime}, G_{1}^{\prime \prime} \in \mathscr{B}_{n, g}^{2}$ be defined as $G_{1}=G_{0}(u) S_{n-r-s-t-1}$, $G_{1}^{\prime}=G_{0}^{\prime}(u) S_{n-g-t+1}$ and $G_{1}^{\prime \prime}=G_{0}^{\prime \prime}(u) S_{n-g}$, where $G_{0}=\theta(r, s, t)$, $G_{0}^{\prime}=\theta(1, g-3, t), G_{0}^{\prime \prime}=\theta(1, g-3,1)$, as given in Figure 5. Then:

(i) $G_{0}$ with a vertex of degree 2 deleted is strictly $m$-greater than $G_{0}$ with a vertex of degree 3 deleted;

(ii) for any $G \in \mathscr{B}_{n, g}^{2}$ with $\theta(r, s, t)$ as its brace, $G \succeq G_{1}$ with equality if and only if $G \cong G_{1}$;

(iii) $G_{1} \succeq G_{1}^{\prime}$ with equality if and only if $G_{1} \cong G_{1}^{\prime}$;

(iv) $G_{1}^{\prime} \succeq G_{1}^{\prime \prime}$ with equality if and only if $G_{1}^{\prime} \cong G_{1}^{\prime \prime}$.

Consequently, for any $G \in \mathscr{B}_{n, g}^{2}, G \succeq G_{1}{ }^{\prime \prime}$ with equality if and only if $G \cong G_{1}^{\prime \prime}$.

\section{Proof.}

(i) Choose two vertices of degrees 2 and 3 respectively in $G_{0}$, say $w_{m}$ and $u$. Consider $G_{0}-w_{m}$, which can be viewed as a cycle $C_{g}$ together with two pendant paths, namely, $P=u w_{1} \cdots w_{m-1}$ at $u$ and $Q=v w_{t} \cdots w_{m+1}$ at $v$, possibly of length 0 . If one of the two paths $P$ or $Q$ is of length 0 , then we can choose an appropriate edge $e$ such that $G_{0}-w_{m}-e \cong P_{n-1}$. Since $P_{n-1}$ is $m$-greater than any tree $T$ of order $n-1$, we have $G_{0}-w_{m} \succ P_{n-1} \succeq G_{0}-u$ as $G_{0}-u$ is a tree.

Now assume that both $P$ and $Q$ are not of length 0. By Lemma 2.1, we have

$$
\begin{aligned}
m_{k}\left(G_{0}-w_{m}\right)-m_{k}\left(G_{0}-u\right)= & m_{k}\left(G_{0}-w_{m}-u w_{1}\right) \\
& +m_{k-1}\left(G_{0}-w_{m}-u-w_{1}\right) \\
& -m_{k}\left(G_{0}-u-w_{m-1} w_{m}\right) \\
& -m_{k-1}\left(G_{0}-u-w_{m-1}-w_{m}\right) .
\end{aligned}
$$

Note that

$$
G_{0}-w_{m}-u-w_{1} \text { and } \quad G_{0}-u-w_{m-1}-w_{m}
$$

are the union of the same graph $T_{g+t-m}-w_{m}$ (see Figure 5 ) and the path $P_{m-2}$ and so are isomorphic. Thus, $m_{k-1}\left(G_{0}-w_{m}-u-\right.$ $\left.w_{1}\right)=m_{k-1}\left(G_{0}-u-w_{m-1}-w_{m}\right)$ for any $k$. It is clear that $G_{0}-w_{m}-u w_{1}$ is the union of a graph $P_{t-m+1}(v) C_{g}$ and a path $P_{m-1}$, and $G_{0}-u-w_{m-1} w_{m}$ is the union of a graph $T_{g+t-m}$ and a path 


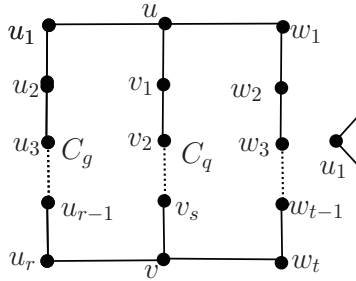

$G_{0}$

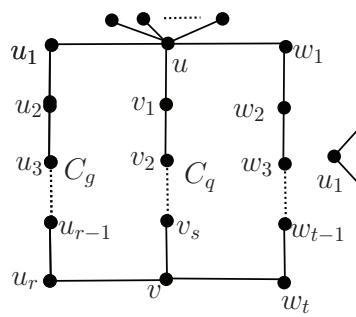

$G_{1}$

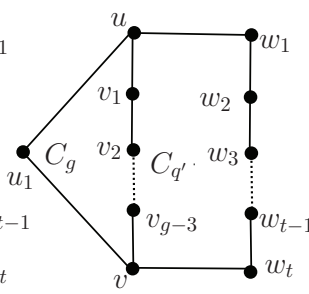

$G_{0}^{\prime}$

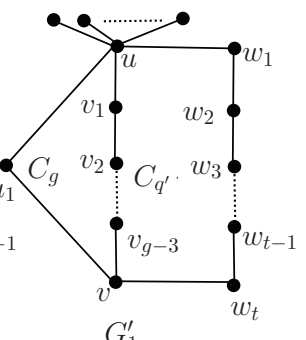

$G_{1}^{\prime}$
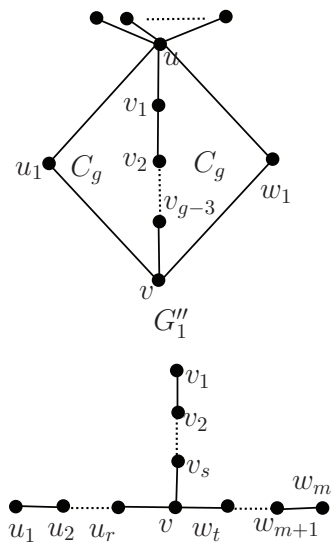

$T_{g+t-m}$

Figure 5.

$P_{m-1}$. Due to $P_{t-m+1}(v) C_{g} \succ P_{r+s+t-m+2} \succ T_{g+t-m}$ and Lemma 2.4, we have $G_{0}-w_{m}-u w_{1} \succ P_{r+s+t-m+2} \cup P_{m-1} \succ G_{0}-u-w_{m-1} w_{m}$. Therefore, $G_{0}-w_{m} \succ G_{0}-u$.

(ii) As in the proof of Theorem 3.1, by Lemma 2.6, we can assume that all tree branches at the cycles of $G$ are stars. Without loss of generality, suppose that $G$ is the coalescence of the vertex $t_{i}$ (here we use new notation for these vertices) in $G_{0}$ and the center of $S_{r_{i}+1}$ for $i=1, \ldots, l$, and $\sum_{i=1}^{l} r_{i}=a$. For convenience, we use $H_{i}$ to denote graphs defined recursively as follows. Let $H_{0}=G_{0}$, and if $H_{i-1}$ is already defined, then $H_{i}$ is defined to be $H_{i-1}\left(t_{i}\right) S_{r_{i}+1}$. Note that $H_{l}=G$.

Applying Lemma 2.2, we have

$$
m_{k}\left(G_{1}\right)=m_{k}\left(G_{0}\right)+a m_{k-1}\left(G_{0}-u\right)
$$

and 


$$
\begin{aligned}
m_{k}(G)= & m_{k}\left(H_{l-1}\right)+r_{l} m_{k-1}\left(H_{l-1}-t_{l}\right) \\
= & m_{k}\left(H_{l-2}\right)+r_{l-1} m_{k-1}\left(H_{l-2}-t_{l-1}\right)+r_{l} m_{k-1}\left(H_{l-1}-t_{l}\right) \\
& +\cdots \\
= & m_{k}\left(G_{0}\right)+\sum_{i=1}^{l} r_{i} m_{k-1}\left(H_{i-1}-t_{i}\right) .
\end{aligned}
$$

First note that $G_{0}-t_{i}$ is a (proper) subgraph of $H_{i}-t_{i}$, and so $H_{i}-t_{i} \succeq G_{0}-t_{i}$. Further, by (i) above, we have $G_{0}-t_{i} \succ G_{0}-u$. Therefore, $H_{i}-t_{i} \succ G_{0}-t_{i} \succ G_{0}-u$ and then $G \succ G_{1}$ unless $G$ is already such a graph $G_{1}$.

(iii) By Corollary 2.12, we have $G_{0} \succeq G_{0}^{\prime}$. Note that $G_{0}-u \cong$ $P_{r+s+1}\left(u_{r+1}, v\right) P_{t}$ and $G_{0}^{\prime}-u \cong P_{r+s+1}\left(u_{2}, v\right) P_{t}$. By Lemma 2.9, we get $G_{0}-u \succeq G_{0}^{\prime}-u$. So we have

$$
\begin{aligned}
m_{k}\left(G_{1}\right) & =m_{k}\left(G_{0}\right)+a m_{k-1}\left(G_{0}-u\right) \\
& \geq m_{k}\left(G_{0}^{\prime}\right)+a m_{k-1}\left(G_{0}^{\prime}-u\right) \\
& =m_{k}\left(G_{1}^{\prime}\right) .
\end{aligned}
$$

Therefore, $G_{1} \succeq G_{1}^{\prime}$. From the process above, equality holds only if $G_{1} \cong G_{1}^{\prime}$.

(iv) By Lemma 2.1,

$$
m_{k}\left(G_{1}^{\prime}\right)=m_{k}\left(G_{1}^{\prime}-v w_{t}\right)+m_{k-1}\left(G_{1}^{\prime}-v-w_{t}\right)
$$

and

$$
m_{k}\left(G_{1}^{\prime \prime}\right)=m_{k}\left(G_{1}^{\prime \prime}-v w_{1}\right)+m_{k-1}\left(G_{1}^{\prime \prime}-v-w_{1}\right) .
$$

By Lemma 2.9, we get $G_{1}^{\prime}-v w_{t} \succeq G_{1}^{\prime \prime}-v w_{1}$ and $G_{1}^{\prime}-v-w_{t} \succeq G_{1}^{\prime \prime}-$ $v-w_{1}$. Hence, $G_{1}^{\prime} \succ G_{1}^{\prime \prime}$ with equality if and only if $G_{1}^{\prime} \cong G_{1}^{\prime \prime}$.

Next, we shall compare the minimal graphs from $\mathscr{B}_{n, g}^{1}$ and $\mathscr{B}_{n, g}^{2}$.

\section{Theorem 3.3.}

$$
S_{n}(g, g) \succ S_{n-g}(u) \theta(1, g-3,1)\left(=G_{1}^{\prime \prime}\right)
$$

(see Figure 5). 
Proof. Let $u_{0}$ be the common vertex of the two copies of $C_{g}$ in $S_{n}(g, g)$, where $G_{1}{ }^{\prime \prime}=S_{n-g}(u) \theta(1, g-3,1)$, and let $u_{1} u_{2}$ be an edge with $u_{1}$ adjacent to $u_{0}$. Note that $S_{n}(g, g)-u_{1} u_{2}$ is the coalescence of a vertex in $C_{g}$ with the center of a star and an end vertex of a path, i.e.,

$$
S_{n}(g, g)-u_{1} u_{2} \cong P_{g-1}\left(u_{0}\right) C_{g}\left(u_{0}\right) S_{n+3-2 g} .
$$

Similarly,

$$
S_{n}(g, g)-u_{1}-u_{2} \cong P_{g-2}\left(u_{0}\right) C_{g}\left(u_{0}\right) S_{n+2-2 g},
$$

and let $u_{1}^{\prime} u_{0}$ be an edge of $C_{g}$ in $S_{n}(g, g)-u_{1}-u_{2}$,

$$
S_{n}(g, g)-u_{1}-u_{2}-u_{1}^{\prime} u_{0} \cong P_{g}\left(u_{0}\right) S_{n+2-2 g}\left(u_{0}\right) P_{g-2} .
$$

It is obvious that $S_{n}(g, g)-u_{1}-u_{2} \succ S_{n}(g, g)-u_{1}-u_{2}-u_{1}^{\prime} u_{0}$. In the same way, we have

$$
G_{1}{ }^{\prime \prime}-v w_{1} \cong C_{g}(u) S_{n-g+1} \text { and } G_{1}^{\prime \prime}-v-w_{1} \cong P_{g-2}(u) S_{n-g+1} \text {. }
$$

By Lemma 2.1, we have

$$
\begin{aligned}
m_{k}\left(G_{1}^{\prime \prime}\right) & =m_{k}\left(G_{1}{ }^{\prime \prime}-v w_{1}\right)+m_{k-1}\left(G_{1}^{\prime \prime}-v-w_{1}\right) \\
& =m_{k}\left(C_{g}(u) S_{n-g+1}\right)+m_{k-1}\left(P_{g-2}(u) S_{n-g+1}\right),
\end{aligned}
$$

and

$$
\begin{aligned}
m_{k}\left(S_{n}(g, g)\right)= & m_{k}\left(S_{n}(g, g)-u_{1} u_{2}\right)+m_{k-1}\left(S_{n}(g, g)-u_{1}-u_{2}\right) \\
= & m_{k}\left(P_{g-1}\left(u_{0}\right) C_{g}\left(u_{0}\right) S_{n+3-2 g}\right) \\
& +m_{k-1}\left(P_{g-2}\left(u_{0}\right) C_{g}\left(u_{0}\right) S_{n+2-2 g}\right) .
\end{aligned}
$$

By Lemma 2.9, we get

$$
P_{g-1}\left(u_{0}\right) C_{g}\left(u_{0}\right) S_{n+3-2 g} \succeq C_{g}(u) S_{n-g+1} .
$$

Choosing an appropriate edge $e$, we have

$$
P_{g-2}\left(u_{0}\right) C_{g}\left(u_{0}\right) S_{n+2-2 g}-e \cong P_{g}\left(u_{0}\right) S_{n+2-2 g}\left(u_{0}\right) P_{g-2},
$$

and again, by Lemma 2.9 ,

$$
P_{g}\left(u_{0}\right) S_{n+2-2 g}\left(u_{0}\right) P_{g-2} \succeq P_{g-2}(u) S_{n-g+1} .
$$

Thus, $S_{n}(g, g) \succ G_{1}{ }^{\prime \prime}$.

From Theorems 3.1, 3.2 and 3.3, we obtain the following main result. 
Theorem 3.4. For any graph $G \in \mathcal{B}_{n, g}$, we have

$$
G \succeq S_{n-g}(u) \theta(1, g-3,1),
$$

and therefore,

$$
\operatorname{ME}(G) \geq \operatorname{ME}\left(S_{n-g}(u) \theta(1, g-3,1)\right),
$$

where equality holds if and only if $G \cong S_{n-g}(u) \theta(1, g-3,1)$.

\section{REFERENCES}

1. L. Chen, J. Liu and Y. Shi, Matching energy of unicyclic and bicyclic graphs with a given diameter, Complexity 21 (2015), 224-238.

2. L. Chen and Y. Shi, The maximal matching energy of tricyclic graphs, MATCH Comm. Math. Comp. Chem. 73 (2015), 105-120.

3. D.M. Cvetković, M. Doob, I. Gutman and A. Torgašev, Recent results in the theory of graph spectra, North-Holland, Amsterdam, 1988.

4. H. Deng, The smallest Hosoya index in $(n, n+1)$-graphs, J. Math. Chem. 43 (2008), 119-133.

5. I. Gutman, The matching polynomial, MATCH Comm. Math. Comp. Chem. 6 (1979), 75-91.

6. I. Gutman and D.M. Cvetković, Finding tricyclic graphs with a maximal number of matchings-Another example of computer aided research in graph theory, Publ. Inst. Math. Nouv. 35 (1984), 33-40.

7. I. Gutman and S. Wagner, The matching energy of a graph, Discr. Appl. Math. 160 (2012), 2177-2187.

8. I. Gutman and F. Zhang, On the ordering of graphs with respect to their matching numbers, Discr. Appl. Math. 15 (1986), 25-33.

9. G. Huang, M. Kuang and H. Deng, Extremal graph with respect to matching energy for a random polyphenyl chain, MATCH Comm. Math. Comp. Chem. $\mathbf{7 3}$ (2015), 121-131.

10. S. Ji, X. Li and Y. Shi, Extremal matching energy of bicyclic graphs, MATCH Comm. Math. Comp. Chem. 70 (2013), 697-706.

11. S. Ji and H. Ma, The extremal matching energy of graphs, Ars Combin. 115 (2014), 343-355.

12. S. Ji, H. Ma and G. Ma, The matching energy of graphs with given edge connectivity, J. Inequal. Appl. 1 (2015), 1-9.

13. X. Li, Y. Shi and I. Gutman, Graph energy, Springer, New York, 2012.

14. X. Li, Y. Shi, M. Wei and J. Li, On a conjecture about tricyclic graphs with maximal energy, MATCH Comm. Math. Comp. Chem. 72 (2014), 183-214.

15. H. Li, B. Tan and L. Su, On the signless Laplacian coefficients of unicyclic graphs, Linear Alg. Appl. 439 (2013), 2008-2009. 
16. S. Li and W. Yan, The matching energy of graphs with given parameters, Discr. Appl. Math. 162 (2014), 415-420.

17. H. Li, Y. Zhou and L. Su, Graphs with extremal matching energies and prescribed parameters, MATCH Comm. Math. Comp. Chem. 72 (2014), 239-248.

18. R. Sun, Z. Zhu and L. Tan, On the Merrifield-Simmons index and Hosoya index of bicyclic graphs with a given girth, Ars Combin. 103 (2012), 465-478.

19. K. Xu, K.C. Das and Z. Zheng, The minimal matching energy of $(n, m)$ graphs with a given matching number, MATCH Comm. Math. Comp. Chem. 73 (2015), 93-104.

College of Mathematics and Information Science, Jiangxi Normal UniverSity, NANCHANG, 330022, China

Email address: lhh@jxnu.edu.cn

College of Mathematics and Information Science, Jiangxi Normal UniverSiTy, NANCHANG, 330022, ChinA

Email address: 105375147@qq.com 